\title{
Moderne Mobilitätsformen und die Bedürfnisse der Gesellschaft
}

\author{
Meike Jipp und Karsten Lemmer
}

\section{Zusammenfassung}

Menschen überwinden räumliche Distanz virtuell mithilfe von Informationsund Kommunikationstechnologien oder physisch unter Nutzung von Verkehrsmitteln wie z. B. Kraftfahrzeugen oder Bussen des öffentlichen Personennahverkehrs. Automatisierung, Digitalisierung und künstliche Intelligenz ermöglichen die Bereitstellung neuer und innovativer Lösungen insbesondere für die Realisierung physischer Mobilität. Ein Beispiel hierfür ist ein bedarfsorientierter, autonomer Shuttle des öffentlichen Personennahverkehrs. Im Rahmen dieses Buchkapitels wird erläutert, dass sich Menschen nur dann für die Nutzung solche Systeme entscheiden, wenn diese Systeme in der Lage sind, menschliche Bedürfnisse nach Sicherheit, sozialen Kontakten oder Selbstverwirklichung zu stillen. Es wird dargestellt, welche (mobilitätsfremden) Services für die Bedürfnisbefriedigung in die Shuttle integriert werden können. Abschließend wird diskutiert, wie eine maßgeschneiderte Mobilitätslösung für die Gesellschaft entstehen kann, die Mobilitäts-, Fahrzeug- und Servicekonzept integrativ verknüpft, sodass Menschen bereit sind, auf ihr individuelles Fahrzeug zu verzichten, die Flächeninanspruchnahme des Verkehrs sinkt und insbesondere Städte lebenswerter werden.

\footnotetext{
M. Jipp $(\bowtie)$

Institut für Verkehrssystemtechnik, Deutsches Zentrum für Luft- und Raumfahrt (DLR) e. V., Braunschweig, Deutschland

E-Mail: Meike.Jipp@dlr.de

K. Lemmer

Deutsches Zentrum für Luft- und Raumfahrt (DLR) e. V., Köln, Deutschland E-Mail: Karsten.Lemmer@dlr.de

(C) The Author(s) 2021 


\section{Schlüsselwörter}

Automatisierung $\cdot$ Digitalisierung $\cdot$ Sicherheit $\cdot$ Mobility-as-a-Service $($ MAAS) $\cdot$ virtuelle Mobilität $\cdot$ ÖPNV $\cdot$ Shuttle $\cdot$ Nutzung $\cdot$ Mensch

\section{$1 \quad$ Einleitung}

Mobilität ist ein Phänomen der Moderne (Lash und Urry 1994). Sie beschreibt Bewegung und Veränderung in den unterschiedlichsten Aspekten der Gesellschaft. Es ist daher auch nicht überraschend, dass Menschen auf die unterschiedlichste Art und Weise mobil sein können (Bonß und Kesselring 1999): Sie bewegen sich räumlich zum Beispiel von ihrer Arbeitsstätte zu ihrem Wohnort und zurück (vgl. Tully und Baier 2006). Menschen bewegen sich aber auch zwischen sozialen Positionen (vgl. Fuchs-Heinritz et al. 2011). So können Menschen zum Beispiel in der beruflichen Hierarchie aufsteigen oder ihren Arbeitgeber wechseln und dabei auf einer vergleichbaren Hierarchieebene bleiben. Menschen können weiter zeitlich mobil sein und zum Beispiel Arbeitszeitmodelle veränderten Lebensbedingungen anpassen (Bonß und Kesselring 1999). Diese Formen der räumlichen, sozialen und zeitlichen Mobilität führen zu einer Flexibilisierung der gesellschaftlichen Strukturen, die die Wissenschaft mithilfe der Implementierung und Evaluation von Maßnahmen zur Mobilitätsumsetzung aktiv mitgestalten kann. Im folgenden Buchkapitel liegt der Fokus auf der Analyse und der Gestaltung räumlicher Mobilität insbesondere aus Sicht der Menschen. Es wird analysiert, warum Menschen mobil sind und wie diese Bedürfnisse nach Mobilität gestillt werden können.

\section{Räumliche Mobilität}

Die räumliche Mobilität beschreibt die virtuelle oder physische Überwindung von räumlicher Distanz (vgl. Zimmermann 2001; Zoche et al. 2002). Beide Formen der Mobilität sowie deren Nutzung werden im Folgenden beschrieben.

\subsection{Virtuelle Mobilität}

Die virtuelle Mobilität überwindet Distanzen ohne physische Bewegung. Virtuell mobil sind Menschen zum Beispiel, wenn 
- sie an verschiedenen Orten sind, sich aber im Rahmen von Online-Meetings in einem virtuellen Raum treffen,

- sie in einem virtuellen Raum Produkte im Rahmen von TeleshoppingAngeboten auswählen, oder

- sich Bankkunden und deren Kundenberater virtuell beim Telebanking treffen und sich zum Beispiel über Anlagemöglichkeiten austauschen

(vgl. Rothengatter 1995). Der physische Ort, an dem sich Menschen befinden, ist irrelevant (Popitz 1989).

Virtuelle Mobilität wird inzwischen durch die Nutzung moderner Informations- und Kommunikationstechnologien ermöglicht (vgl. Scheer et al. 2003). Während die zweite Mobilfunkgeneration Global System for Mobile Communication (GSM) 1985 eine Datenübertragungsrate von 9,6 kBit/s erreichte, können heute mithilfe des Mobilfunkstandards Long Term Evolution (LTE) Datenraten von bis zu $500 \mathrm{MBit} / \mathrm{s}$ übertragen werden (Sauter 2013). Hiermit können Gesprächspartner virtuell in den eigenen Raum eingeblendet werden, sodass sich virtuelle und tatsächliche Realität überlagern (Popitz 1989). Solche Technologien zur Generierung von virtuellen Realitäten, aber auch ChatProgramme, Textnachrichten, Emails und Internet-Telefonie ggf. mit Videoübertragung, werden immer öfter herangezogen werden, um in fast allen Bereichen des Lebens mobil zu sein (Popitz 1989; Zoche et al. 2002): Freizeit, Alltagsorganisation, Bildung und Weiterbildung, soziale Interaktion sowie Arbeit. Lediglich für die Gesundheitsversorgung von Patienten wird diese Form der Mobilität kaum genutzt. Zusammenfassend stellt also die virtuelle Mobilität eine gesellschaftlich akzeptierte Form der Beweglichkeit dar (vgl. Harnischfeger et al. 1998), die allerdings die physische Mobilität nicht ersetzt, sondern diese lediglich erweitert (Rothengatter 1995; Zoche et al. 2002).

\subsection{Physische Mobilität}

Physische Mobilität bezeichnet die tatsächliche Überwindung von räumlicher Distanz (vgl. Zoche et al. 2002). Diese Form der Mobilität nutzen jeden Tag ca. $85 \%$ der deutschen Bevölkerung, um - je nach Alter und Geschlecht - einer Arbeit nachzugehen, Einkäufe zu erledigen, sich zu bilden oder sich weiterzubilden, Freizeitaktivitäten nachzugehen oder andere Menschen zu begleiten (MiD 2018). In Deutschland legt dabei jede Person durchschnittlich 3,1 Wege zurück und überwindet eine räumliche Distanz von rund $39 \mathrm{~km}$. Diese Mobilität wird - 
mit Ausnahme von Bewegungen zu Fuß - mit Hilfe von Verkehrsmitteln realisiert (vgl. Ammoser und Hoppe 2006). Klassische Beispiele für Verkehrsmittel sind

- Fahrzeuge, von denen in jedem deutschen Haushalt ca. 1,4 Stück zur Verfügung stehen und mit denen durchschnittlich entweder ca. $43 \%$ (Fahrer) bzw. ca. $14 \%$ (Mitfahrer) der Wege in Deutschland zurückgelegt werden,

- Fahrräder, von denen fast jeder Bewohner in Deutschland eines besitzt und mit denen durchschnittlich ca. $11 \%$ der Wege in Deutschland zurückgelegt werden und

- Busse, Straßenbahnen, Züge, mit denen durchschnittlich $10 \%$ der Wege in Deutschland zurückgelegt werden (MiD 2018).

Die restlichen $22 \%$ der Wege werden zu Fuß zurückgelegt (MID 2018).

Veränderungen in der physischen Mobilität lassen sich in zwei Kategorien einteilen: Einerseits entstehen neue Verkehrsmittel. Hierzu zählen zum Beispiel

- elektrische Scooter, bei denen der Fahrer auf einem Trittbrett zwischen zwei hintereinander angeordneten Rädern steht, welches mithilfe einer Lenkstange gesteuert wird, und deren westeuropäischer Markt gemäß Prognosen bis 2025 um $15 \%$ wachsen wird (vgl. Andrada et al. 2016; Hardt und Bogenberger 2017) und

- Segways, bei denen der Fahrer auf einer Plattform zwischen zwei nebeneinander angeordneten Rädern steht, die durch eine Gewichtsverlagerung des Fahrers gesteuert werden und die sich inzwischen insbesondere für bestimmte Personengruppen wie Polizisten und Personen mit Mobilitätseinschränkungen als Verkehrsmittel durchgesetzt haben (vgl. Sawatzky et al. 2007).

Diese neuen Verkehrsmittel substituieren - ähnlich wie virtuelle Mobilitätsangebote - die traditionellen Verkehrsmittel nicht, sondern ergänzen diese lediglich.

Andererseits ermöglichen Technologien wie Automatisierung, Digitalisierung und künstliche Intelligenz eine Veränderung klassischer Verkehrsmittel (vgl. Perret et al. 2018). So werden zum Beispiel

- automatisierte Kraftfahrzeuge, die - je nach Automatisierungsgrad Menschen die Fahrzeugsteuerung abnehmen (Bundesanstalt für Straßenwesen 2012), 
- Leihräder, mit denen Personen kurze Strecken zumeist im urbanen Umfeld durchführen können und deren Verfügbarkeit und Nutzung in den letzten Jahren in Europa exponentiell gewachsen sind (Böhm et al. 2015; Parkes et al. 2013),

- Ridesharing-Angebote, bei denen Privatpersonen anderen Personen anbieten, sie zu einem ähnlichen Zeitpunkt zu einem ähnlichen Ziel zu fahren (vgl. Agatz et al. 2012),

- Ridepooling-Angebote, bei denen Personen mit Personenbeförderungsschein mehrere Kunden gemeinsam zu einem Ziel fahren (vgl. Perret et al. 2018) und

- bedarfsorientierter öffentlicher Personennahverkehr (ÖPNV), der Kunden zeitlich und geographisch flexibel befördert und in den traditionellen ÖPNV integriert ist (vgl. König et al. 2018),

zur Verfügung gestellt. Dank künstlicher Intelligenz können insbesondere die Flexibilisierung der Route und des Zeitplans sowie die Fahrzeugautomatisierung kombiniert werden: So werden derzeit Mobilitätsformen entwickelt, bei denen Kunden mit automatisierten Fahrzeugen bedarfsorientiert, also ohne vorab definierten Zeitplan und Route an ihren Zielort transportiert werden (vgl. Dreßler et al. 2018).

\section{Evaluation der Wirksamkeit räumlicher Mobilitätsformen}

\subsection{Metriken}

Effekte räumlicher Mobilitätsformen können mithilfe verschiedener Metriken beschrieben werden. Diese Metriken erlauben den Vergleich verfügbarer Mobilitätsformen (vgl. Umweltbundesamt 2012):

- Sicherheit beschreibt die Abwesenheit von Situationen, die Menschenleben gefährden (vgl. BMVI 2011).

- Umgebungslärm beschreibt die Belastung, die u. a. durch Mobilität entsteht. Er erhöht bei Menschen das Risiko von Erkrankungen des Herz-Kreislauf-Systems (vgl. Heinrichs et al. 2011).

- Emissionen beschreiben den Ausstoß von Treibhausgasen, Stickstoffoxiden und Feinstaub (vgl. Umweltbundesamt 2012).

- Der Flächenverbrauch beschreibt die Fläche, die Verkehrsmittel in Anspruch nehmen (vgl. Umweltbundesamt 2012). 
Vergleicht man zum Beispiel traditionelle Verkehrsmittel bzgl. des Flächenverbrauchs, so zeigt sich, dass ein stillstehendes, privates Kraftfahrzeug eine Fläche von ca. 13,5 $\mathrm{m}^{2}$ in Anspruch nimmt. Dieser Flächenverbrauch liegt bei einem Omnibus, der zu $20 \%$ besetzt ist, bei $2,5 \mathrm{~m}^{2}$ und bei einem Omnibus, der zu $40 \%$ besetzt ist, bei $1,2 \mathrm{~m}^{2}$ (Umweltbundesamt 2012). Diese Flächen werden aktuell für Verkehr frei gehalten und verhindern somit die alternative Nutzung der Flächen für Grünanlagen oder Erholungsgebiete (Randelhoff 2014). Städte könnten also attraktiver gestaltet werden (vgl. Greenberg 1996), wenn die Flächeninanspruchnahme für Verkehr sinkt bzw. wenn Menschen auf ihr individuelles Fahrzeug verzichten und Angebote des ÖPNV nutzen, da dieser weniger Fläche verbraucht.

\subsection{Mobilität zur Bedürfniserfüllung}

Menschen sind insbesondere in ihrer Rolle als Verkehrsteilnehmer keine Beförderungsobjekte, sondern entscheidungsfähige und aktiv handelnde Individuen (vgl. Hildebrandt et al. 2001). Sie entscheiden selbst, welches Verkehrsmittel für sie geeignet ist, um Mobilitätsziele zu erreichen. Die Wahl fällt dabei, wie Abschn. 2.2 zeigt, häufig auf individuelle Fahrzeuge. Menschen betrachten diese Fahrzeuge also als geeignet für ihre Bedürfnisbefriedigung. Bedürfnisse sind Motivatoren, die eine grundlegende Antriebskraft für Menschen sind und den Fokus menschlicher Aufmerksamkeit darauf richten, einen Ist- in einen gewünschten Soll-Zustand zu überführen (vgl. Asendorpf 2005; Disse 2016). Bedürfnisse treiben somit menschliches Verhalten an und zeigen, was Menschen zum Erhalt und zur Entfaltung ihres Lebens benötigen (Lederer 1980).

Zahlreiche Bedürfnistheorien zeigen, welche Bedürfnisse menschliches Verhalten antreiben (vgl. Lewin 1926; McClelland 1985; Maslow 1954). So ordnete zum Beispiel Maslow (1954) Bedürfnisse in einer sogenannten Bedürfnishierarchie: Demnach streben Menschen zunächst danach, Defizitbedürfnisse und anschließend Wachstumsbedürfnisse zu stillen. Defizitbedürfnisse entstehen durch einen Mangel an Reizen. Bei Wachstumsbedürfnissen steht das Streben nach Reizen im Vordergrund. Maslow (1954) ordnete auch die Defizitbedürfnisse in eine Reihenfolge: Menschen stillen zunächst primäre physiologische Bedürfnisse (Hunger, Durst, Sauerstoff). Danach stehen Sicherheitsbedürfnisse (Freiheit von Angst, Behaglichkeit) und soziale Bedürfnisse (Anerkennung, Vertrauen) im Fokus. Zuletzt konzentrieren sich Menschen darauf, ihr Geltungsbedürfnis zu befriedigen. Sind diese Defizitbedürfnisse gestillt, streben Menschen nach Selbstverwirklichung - dem einzigen Wachstumsbedürfnis, 
welches Maslow (1954) propagierte. Die Gründe, warum Menschen physisch und virtuell mobil sind (s. auch Abschn. 1), lassen sich genau in diese Bedürfnishierarchie einordnen:

- Die Erledigung von Einkäufen dient teilweise der Befriedigung von primären, physiologischen Bedürfnissen.

- Das Streben nach Bildung und Weiterbildung kann als Maßnahme zur Befriedigung nach Geltung gesehen werden.

- Die Begleitung anderer Menschen kann dazu dienen, soziale Bedürfnisse zu stillen.

- Freizeitaktivitäten dienen sicherlich der Selbstverwirklichung.

Satisfaktoren sind die Mittel, die Menschen heranziehen, um Bedürfnisse zu stillen (vgl. Cruz et al. 2009; Mallmann 1980). Hierbei können primäre von sekundären Satisfaktoren unterschieden werden: Primäre Satisfaktoren sind Arbeitsmittel, die die Bedürfnisbefriedigung direkt ermöglichen. So sind Lebensmittel primäre Satisfaktoren zur Befriedigung des Bedürfnisses nach Nahrung. Sekundäre Satisfaktoren sind hingegen Mittel zum Zweck. So ermöglicht zum Beispiel Geld das Einkaufen von Lebensmitteln, mit denen dann wiederum das Bedürfnis nach Nahrung gestillt werden kann. Geld dient in diesem Beispiel also als sekundärer Satisfaktor. Wird dieses Konzept auf Mobilität übertragen, dann sind Verkehrsmittel Satisfaktoren. Menschen wählen das Verkehrsmittel aus, mithilfe dessen die eigenen Bedürfnisse am besten - primär oder sekundär - gestillt werden können. Nach aktuellen Statistiken ist dies oftmals das individuelle Fahrzeug.

Sollten nun Menschen eher innovative ÖPNV-Angebote nutzen, die weniger Fläche beanspruchen, sollte untersucht werden, ob diese Angebote die menschliche Bedürfnisbefriedigung (primär oder sekundär) ermöglichen. Grippenkoven et al. (2018) untersuchten daher die Nutzungsbereitschaft von bedarfsorientierten und autonomen ÖPNV-Shuttles. Im Rahmen von Workshops baten die Autoren Probanden, einen autonomen ÖPNV-Shuttle zu nutzen, der allerdings noch nicht bedarfsorientiert eingesetzt wird. Die Probanden sollten sich vorstellen, wie das System wirkt, wenn es nicht nach Zeitplan auf einer festgelegten Route unterwegs ist. Die Probanden arbeiteten Nutzungshemmnisse heraus, die die Autoren mit Hilfe von qualitativen Inhaltsanalysen in folgende Kategorien von Nutzungshemmnissen einteilen konnten:

- Angst vor anderen Menschen: Menschen fürchteten kritische Gruppendynamiken, andere Passagiere, die sich im Bus merkwürdig benehmen, physische Gewalt, Diebstahl, Vandalismus, überfüllte Transportmittel und Mitmenschen, die nicht bereit sind, zu helfen, sollte dies notwendig werden. 
- Ängste vor einem intransparenten System: Menschen fürchteten, dass der Shuttle unerwartet zum Halten kommt, dass der Shuttle unpünktlich ist, dass er eine unbekannte Route fährt und dass nicht klar wird, ob es ein mögliches Hindernis korrekt erkannt hat oder nicht.

- Ängste vor nicht-funktionsfähiger Technologie: Menschen fürchteten, dass das System schlecht/nicht gewartet ist, dass die eigenen Daten nicht sicher sind und dass sie das System nicht durch einen Notausgang verlassen können, sollte dies notwendig werden.

Werden diese Kategorien vor dem Hintergrund der Bedürfnishierarchie von Maslow (1954) analysiert, wird deutlich, dass die skizzierten autonomen und bedarfsorientierten ÖPNV-Shuttles das menschliche Bedürfnis nach Sicherheit zumindest in der untersuchten Stichprobe nicht ausreichend stillen konnten. Es ist daher auch zu erwarten, dass ein solches Verkehrssystem Menschen kaum davon überzeugen wird, vollständig auf ihr individuelles Fahrzeug zu verzichten, zumindest nicht in der skizzierten Form.

\section{$4 \quad$ Bedürfnisorientierte Gestaltung von Mobilitätsangeboten}

Mobilitätsangebote sollten so gestaltet werden, dass sie idealerweise die primäre und sekundäre Bedürfnisbefriedigung der Menschen erlauben. Wie dies erreicht werden kann, zeigten Grippenkoven et al. (2018) am Beispiel des Sicherheitsbedürfnisses bei der Nutzung autonomer und bedarfsorientierter ÖPNV-Shuttles. In Workshops arbeiteten die Probanden Maßnahmen aus, die gegen die vorab definierten Nutzungshemmnisse helfen und das Sicherheitsbedürfnis besser befriedigen sollten. Folgende Maßnahmen schlugen die Probanden vor:

- Individuelle Kabine: Die Probanden wünschten sich eine Kabine innerhalb des Transportvehikels, welches transparente Wände besitzt und von innen abschließbar ist. Diese Kabine sollte für Kunden in Notfallsituationen ein sicherer Raum darstellen.

- Schließsystem: Die Probanden wünschten sich ein Schließsystem für den ÖPNV-Shuttle, sodass dieser während einer Fahrt durch eine schlechte Gegend bei Bedarf von Innen verschlossen und somit verhindert werden kann, dass andere Personen einsteigen. 
- Überwachungssystem für Sitzplätze: Die Probanden wünschten sich eine App, mithilfe derer freie Sitzplätze angezeigt werden können und ein eigener Sitzplatz gebucht werden kann.

- Notbremse: Die Probanden wünschten sich eine Notbremse, die von Passagieren des ÖPNV-Shuttles betätigt werden kann.

- Mobiler Sicherheitsdienst: Die Probanden wünschten sich eine Videoüberwachung des ÖPNV-Shuttles. Mit Hilfe dieser Videoüberwachung sollten Anbieter der ÖPNV-Shuttles kritische Situationen im Fahrzeug identifizieren können und dann bei Bedarf einen mobilen Sicherheitsdienst zum Fahrzeug schicken.

- Videobasiertes Assistenzsystem: Die Probanden wünschten sich Monitore, Kameras, Lautsprecher und Mikrofone im ÖPNV-Shuttle. Mithilfe dieser Technologie sollten Passagiere bei Bedarf Kontakt mit einer Zentrale aufnehmen können.

- Meldesystem: Die Probanden wünschten sich eine App, mithilfe derer Anzeichen von Vandalismus, defekte Installationen oder Schmutz an eine Zentrale gemeldet werden können. Mit diesem Meldesystem sollte außerdem ein Notruf abgesetzt werden können.

- Monitore zur Navigation: Die Probanden wünschten sich außerdem einen Monitor, der die aktuelle Position des ÖPNV-Shuttles, die geplante Route und die Positionen anderer Shuttles in der Nähe anzeigt.

- Training: Die Probanden wünschten sich Informationsveranstaltungen und Trainings darüber, wie das ÖPNV-System genau bedient werden kann und wie es technisch funktioniert.

- Informationssystem: Die Probanden wünschten sich ein Informationssystem, welches im ÖPNV-Shuttle zur Verfügung stehen sollte und Passagiere darüber informiert, wie das System technisch funktioniert.

- Display: Die Probanden wünschten sich ein Display, welches die aktuelle Geschwindigkeit, Fahrtrichtung und erkannte Hindernisse anzeigt.

- Notfallknopf: Die Probanden wünschten sich einen Notfallknopf, mithilfe dessen eine Sprachverbindung zu einem Notfallzentrum aufgebaut wird.

- Telefon: Die Probanden wünschten sich ein Telefon, mithilfe dessen Passagiere, die kein eigenes Mobiltelefon besitzen, andere Menschen über Änderungen der Route oder über die Entwicklung einer kritischen Situation informieren können.

Ob diese Maßnahmen tatsächlich das menschliche Sicherheitsbedürfnis in bedarfsorientierten und autonomen ÖPNV-Shuttles verbessern, untersuchten Grippenkoven et al. (2018) mit Hilfe von Online-Surveys. Die Probanden 
erhielten zunächst eine Einführung in den autonomen, bedarfsorientierten ÖPNV-Shuttle und sollten dann bewerten, wie hoch sie die eigene Sicherheit in einem solchen ÖPNV-Shuttle einschätzen. Weiter wurden die Probanden mit einem Szenario konfrontiert, welches im Rahmen der Workshops als Nutzungshemmnis beschrieben wurde. Die subjektive Sicherheit wurde wiederholt erfasst, sodass inferenzstatistisch analysiert werden konnte, ob die subjektive Sicherheit tatsächlich sinkt. Abschließend bekamen die Probanden eine Einführung in ausgewählte Maßnahmen, die die Sicherheit erhöhen sollten. Durch eine weitere Erfassung der subjektiven Sicherheit konnte nun mithilfe der Daten überprüft werden, ob die Maßnahmen tatsächlich geeignet sind, das menschliche Sicherheitsbedürfnis besser zu stillen. Der Online-Fragebogen war so aufgebaut, dass Daten von insgesamt 106 Personen zu allen Nutzungshemmnissen (s. Abschn. 3.2) und Maßnahmen erfasst wurden. Die Datenanalyse von Grippenkoven et al. (2018) zeigte,

- dass alle Nutzungshemmnisse, die Angst vor anderen Passagieren und vor einem intransparenten System (für Details, s. Abschn. 3.2) darstellten, das subjektive Sicherheitsgefühl signifikant verschlechterten,

- dass insbesondere der fehlende Notausgang als einziges Hemmnis in der Kategorie der nicht-funktionalen Technik das subjektive Sicherheitsgefühl signifikant verschlechterte.

Die Analysen zeigten weiter, dass insbesondere die individuelle Kabine, die Notbremse, der mobile Sicherheitsdienst, das videobasierte Assistenzsystem und der Notfallknopf die subjektive Sicherheit der Personen signifikant erhöhten und gegen fast alle Nutzungshemmnisse halfen (vgl. Grippenkoven et al. 2018). Zusammenfassend scheint also die Implementierung dieser Maßnahmen das Sicherheitsbedürfnis in den autonomen und bedarfsorientierten ÖPNV-Shuttles zu erhöhen, die Attraktivität der ÖPNV-Shuttles als Satisfaktor zu steigern und dazu beizutragen, dass sich Menschen eher für die Nutzung dieser Systeme entscheiden.

Sicherheit ist jedoch nicht das einzige Bedürfnis, dessen Befriedigungswunsch der Nutzung von ÖPNV-Angeboten im Weg stehen kann. Auch die anderen Bedürfnisse, welche Maslow (1954) in seiner Bedürfnishierarchie unterschied, lassen sich unter Umständen über mobilitätsfremde Angebote innerhalb bedarfsorientierter, autonomer ÖPNV-Shuttle stillen:

- Primäre physiologische Bedürfnisse könnten in Einkaufsshuttles, Wellnessshuttles, Ärzteshuttles oder Ruheshuttles gestillt werden. 
- Soziale Bedürfnisse könnten in Spieleshuttles oder Reiseshuttles mit spezifischen Nutzergruppen gestillt werden.

- Das Geltungsbedürfnis könnte in Lern-, Vortrags- oder Orchestershuttles gestillt werden.

- Das Bedürfnis nach Selbstverwirklichung könnte in Kreativitäts- oder Büroshuttles gestillt werden.

Die Umsetzung solcher Angebote in ÖPNV-Shuttles birgt Herausforderungen: So müssen geeignete Service-, Mobilitäts- und Fahrzeugkonzepte integrativ entwickelt werden. Das Servicekonzept beschreibt dabei die Angebote, die - über Mobilität hinaus - in Shuttles integriert werden und relevante menschliche Bedürfnisse einer hinreichend großen Population stillen können. Identifiziert werden können solche Angebote - wie von Grippenkoven et al. (2018) dargestellt - mithilfe einer Verknüpfung von qualitativen und quantitativen Forschungsmethoden. Hiermit können Servicelösungen zusammen mit potenziellen Nutzern erarbeitet und evaluiert werden. Basierend auf diesen Lösungen können dann Anforderungssets an die benötigten Fahrzeug- und Mobilitätskonzepte abgeleitet werden. Das Fahrzeugkonzept beschreibt dabei die grundlegenden Eigenschaften der Vehikel (Achleitner et al. 2007), die für solche Serviceleistungen benötigt werden. Wird zum Beispiel ein Spieleshuttle ins Leben gerufen, sollte sich eine größere Gruppe von Personen gleichzeitig im Shuttle aufhalten können. Auch sollte die Kommunikation und Interaktion innerhalb der Gruppe durch ein geeignetes Fahrzeugkonzept gefördert werden. Wird aber zum Beispiel ein Reiseshuttle benötigt, so sollte das Fahrzeugkonzept auch die Unterbringung von größeren Gepäckmengen berücksichtigen. Es ist also essenziell, modulare Fahrzeugkonzepte zur Verfügung zu stellen, die möglichst flexibel verschiedene Servicekonzepte bedienen können.

Das Mobilitätskonzept beschreibt, wie Verkehr sicher und leistungsfähig umgesetzt und Mobilität für alle garantiert werden kann (vgl. Winkler 2017). Die Nutzung zum Beispiel von Spieleshuttles sollte nicht dem Selbstzweck dienen. Sie sollten dazu beitragen, dass Menschen, wenn sie sich zu einem Zielort begeben, weitere Bedürfnisse stillen können. Das Mobilitätskonzept muss also die Bewegung hin zu einem Zielort unterstützen. Es muss sicherstellen, dass Personen, die ihr soziales Bedürfnis in einem Spieleshuttle stillen wollen, und die zu einem ähnlichen Zeitpunkt zu einem ähnlichen Ziel wollen, gemeinsam befördert werden. Ein solches flexibles Mobilitätskonzept wird auf künstliche Intelligenz aufbauen und zusammen mit Service- und Fahrzeugkonzepten ein maßgeschneidertes Konzept zur Realisierung physischer Mobilität ergeben. Dieses Konzept bietet Menschen die primäre und sekundäre Bedürfnisbefriedigung in Shuttles des öffentlichen Personennah- und -fernverkehrs an. Es kann dazu beitragen, dass 
Menschen eher bereit sind, auf die Nutzung individueller Fahrzeuge zu verzichten, die Flächeninanspruchnahme von Verkehr zu reduzieren und Städte grüner und lebenswerter zu gestalten.

\section{$5 \quad$ Zusammenfassung}

In diesem Buchkapitel wurde Mobilität als Bewegung der Gesellschaft eingeführt. Die räumliche Mobilität kann dabei virtuell mithilfe von Informationsund Kommunikationstechnologien oder physisch mithilfe von Verkehrsmitteln bzw. zu Fuß erfolgen. Die physische Mobilität ist aufgrund von Technologien wie künstlicher Intelligenz, Automatisierung und Digitalisierung starken Veränderungen unterworfen. Einerseits entstehen neue und innovative Verkehrsmittel wie E-Scooter und Segways, die das Erscheinungsbild der Städte immer stärker prägen, aber auch klassische Verkehrsmittel nicht substituieren. Andererseits entstehen innovative Mobilitätsformen. Ein Beispiel hierfür sind automatisierte ÖPNV-Shuttles, die Personen gemeinsam mit anderen Personen zu einem gewünschten Zeitpunkt an einen gewünschten Zielort fahren.

Mobilitätsformen können mit Hilfe von Metriken wie der Flächeninanspruchnahme oder gemäß ihrer Fähigkeit, menschliche Bedürfnisse zu stillen, miteinander verglichen werden. Ein solcher Vergleich zeigt zum Beispiel, dass der Flächenverbrauch individueller Kraftfahrzeuge deutlich höher ist als der des ÖPNV-Systems. Individuelle Kraftfahrzeuge werden aber stärker genutzt. Eine Konsequenz davon ist, dass Städte größere Flächen für Verkehr bereitstellen, als nötig wäre, wenn Menschen eher ÖPNV-Angebote nutzen würden. Insbesondere eine Analyse von Nutzungshemmnissen von bedarfsorientierten, flexiblen ÖPNV-Shuttles zeigte jedoch, dass das menschliche Sicherheitsbedürfnis in diesen Shuttles nicht ausreichend gestillt wird. Mit Hilfe von qualitativen und quantitativen Datenerhebungsmethoden wurden daher Maßnahmen abgeleitet, mithilfe derer das Sicherheitsbedürfnis von potenziellen Nutzern eher befriedigt wird (vgl. Grippenkoven et al. 2018). Darauf aufbauend wurden Services eingeführt, die weitere menschliche Bedürfnisse wie zum Beispiel nach sozialen Kontakten und Selbstverwirklichung im Rahmen der Nutzung von ÖPNV-Shuttles stillen können. Eine Implementierung dieser Services wird möglich, wenn Servicekonzepte intelligent mit Mobilitäts- und Fahrzeugkonzepten verbunden werden. Hieraus können maßgeschneiderte und menschzentrierte Mobilitätslösungen entstehen, die Menschen davon überzeugen könnten, auf ihr individuelles Fahrzeug zu verzichten und stattdessen bedarfsorientierte und autonome ÖPNV-Shuttles zur Umsetzung physischer Mobilität zu nutzen. 


\section{Literatur}

Achleitner, A., Gorissen, W., \& Döllner, G. (2007). Fahrzeugkonzept und Package. In H.-H. Braess \& U. Seiffert (Hrsg.), Vieweg Handbuch Kraftfahrzeugtechnik (S. 93-111). Wiesbaden: Springer.

Agatz, N., Erera, A., Savelsbergh, M., \& Wang, X. (2012). Optimization for dynamic ride-sharing: A review. European Journal of Operational Research, 223(1), 295-303.

Ammoser, H., \& Hoppe, M. (2006). Glossar Verkehrswesen und Verkehrswissenschaften. Dresden: Institut für Wirtschaft und Verkehr.

Andrada, P., Martínez, E., Blanqué, B., Torrent, M., Perat, J. I., \& Sánchez, J. A. (2016). New axial-flux switched reluctance motor for e-scooters. In: Electrical Systems for Aircraft, Railway, Ship Propulsion and Road Vehicles \& International Transportation Electrification Conference, Toulouse, Frankreich: 02.-04.11.2016.

Asendorpf, J. B. (2005). Psychologie der Persönlichkeit. Heidelberg: Springer.

BMVI. (2011). Verkehrssicherheitsprogramm. Berlin: BMVI.

Böhm, O., Grünewald, D., Hertel, M., Smolders, T., \& Zappe, A. (2015). Öffentliche Radvermietsysteme als Ergänzung zum ÖPNV. Überblick über diverse Formen der Trägerschaft. Der Nahverkehr, 10, 32-39.

Bonß, W., \& Kesselring, S. (1999). Mobilität und Moderne: Zur gesellschaftstheoretischen Verortung des Mobilitätsbegriffs. In C. J. Tully (Hrsg.), Erziehung zur Mobilität: Jugendliche in der automobilen Gesellschaft (S. 39-66). Frankfurt a. M.: Campus.

Bundesanstalt für Straßenwesen. (2012). Rechtsfolgen zunehmender Fahrzeugautomatisierung. Bergisch-Gladbach: Bundesanstalt für Straßenwesen.

Cruz, I., Stahel, A., \& Max-Neef, M. (2009). Towards a systematic development approach: Building on the human-scale-development paradigm. Ecological Economics, 68, 20212030 .

Disse, J. (2016). Desiderium. Eine Philosophie des Verlangens. Stuttgart: Kohlhammer.

Dreßler, A., Grippenkoven, J., \& Jipp, M. (2018) Nutzerzentrierte Gestaltung eines Shuttleverkehrs mit autonom fahrenden elektrischen Kleinbussen in der Hamburger Hafencity. VDV-Zukunftskongress Autonomes Fahren im ÖPNV, 21.-22. Juni 2018, Berlin.

Fuchs-Heinritz, W., Klimke, D., Lautmann, R., Rammstedt, O., Staheli, U., Weischer, C., \& Wienold, H. (2011). Lexikon zur Soziologie. Wiesbaden: VS Verlag für Sozialwissenschaften.

Greenberg, K. (1996). Toronto: The urban waterfront as a terrain of availability. In P. Malone (Hrsg.), City, capital, and water (S. 195-218). Oxon: Routledge.

Grippenkoven, J., Fassina, Z., König, A., \& Dressler, A. (2018). Perceived safety: A necessary precondition for successful autonomous mobility services. In D. de Waard (Hrsg.), Proceedings of the human factors and ergonomics society Europe chapter 2018 Annual Conference. Berlin: HFES Europe Chapter.

Hardt, C. \& Bogenberger, K. (2017). Usability of escooters in urban environments: A pilot study. In: IEEE Intelligent Vehicles Symposium, Los Angeles, CA, 11.-14.06.2017.

Harnischfeger, M., Kolo, C., \& Zoche, P. (1998). Medien-Zukunft 2005/2015: Mediennutzung der Zukunft im privaten Sektor. Karlsruhe: Fraunhofer Gesellschaft-ISI. 
Heinrichs, E., Kumsteller, F., Rath, S., Conrad, M., \& Schweigerdt, S. (2011). Lärmbilanz 2010: Untersuchung der Entscheidungskriterien für festzulegende Lärmminderungsmaßnahmen in Lärmaktionsplänen nach der Umgebungslärmrichtlinie 2002/49/EG. Berlin: LK Argus.

Hildebrandt, N., Deubel, K., \& Dick, M. (2001). „Mobilität“: Ein multidisziplinärer Begriff im Alltagsverständnis. Harburger Beiträge zur Psychologie und Soziologie der Arbeit Nr. 23. Hamburg: Technische Universität Hamburg-Harburg.

König, A., Karnahl, K., Gebhardt, L., \& Klötzke, M. (2018). Reallabor Schorndorf: Bedarfsgesteuerte Mobilität gemeinsam gestalten. 12. Deutscher Nahverkehrstag, 24.26.04.2018, Koblenz.

Lash, S., \& Urry, J. (1994). Economics of signs and space. London: Sage.

Lederer, K. (1980). Introduction. In K. Lederer, D. Antal, \& J. Galtung (Hrsg.), Human needs: A contribution to the current debate (S. 3-14). Cambridge: Oelgeschlager.

Lewin, K. (1926). Vorsatz, Wille und Bedürfnis. Mit Vorbemerkungen über die psychischen Kräfte und Energien und die Struktur der Seele. Berlin: Springer

Mallmann, C.A. (1980). Society, needs, and rights: A systematic approach. In: K. Lederer, D. Antal, \& J. Galtung (Hrsg.), Human needs: A contribution to the current debate (S. 37-54). Cambridge: Oelgeschlager.

Maslow, A. H. (1954). Motivation and personality. New York: Harper \& Row.

McClelland, D. C. (1985). How motives, skills, and values determine what people do. American Psychologist, 40, 812-825.

MiD. (2018). Mobilität in Deutschland: Ein Ergebnisbericht. Bonn: BMVI.

Parkes, S. D., Marsden, G., Shaheen, S. A., \& Cohen, A. P. (2013). Understanding the diffusion of public bikesharing systems: Evidence from Europe and North America. Journal of Transport Geography, 31, 94-103.

Perret, F., Fischer, R., \& Frantz, H. (2018). Automatisiertes Fahren als Herausforderung für Städte und Regionen. Zeitschrift für Technikfolgenabschätzung in Theorie und Praxis, 27(2), 31-37.

Popitz, H. (1989). Epochen der Technikgeschichte. Tübingen: Mohr.

Randelhoff, M. (2014). Vergleich unterschiedlicher Flächeninanspruchnahmen nach Verkehrsarten (pro Person). Dresden: Zukunft der Mobilität.

Rothengatter, W. (1995). Mobilität und Kommunikation als Basis dynamischer Wirtschaftsprozesse an den Grenzen physischer Möglichkeiten. In: Fraunhofer-Gesellschaft (Hrsg.), Kommunikation ohne Verkehr? Neue Informationstechniken machen mobil. Tagungsband Fraunhofer Forum 1995, München 1995.

Sawatzky, B., Denison, I., Langrish, S., Richardson, S., Hiller, K., \& Slobogean, B. (2007). The Segway Personal Transporter as an alternative mobility device for people with disabilities: A pilot study. Archives of Physical Medicine and Rehabilitation, 88(11), 1423-1428.

Sauter, M. (2013). Grundkurs Mobile Kommunikationssysteme: UMTS, HSPA und LTE, GSM, GPRS, Wireless WLAN und Bluetooth. Wiesbaden: Springer Vieweg.

Scheer, A. W., Angeli, R., \& Herrmann, K. (2003). Moderne Informations- und Kommunikationstechnologien: Treiber neuer Kooperations- und Kollaborationsformen. In J. Zentes, B. Swoboda, \& D. Morschett (Hrsg.), Kooperationen, Allianzen und Netzwerke (S. 359-384). Wiesbaden: Gabler. 
Tully, C. J., \& Baier, D. (2006). Mobiler Alltag: Mobilität zwischen Option und Zwang: Vom Zusammenspiel biographischer Motive und sozialer Vorgaben. Wiesbaden: VS Verlag für Sozialwissenschaften.

Umweltbundesamt. (2012). Daten zum Verkehr. Dessau-Roßlau: Umweltbundesamt.

Winkler, A. (2017). Mobilität als Gestaltungsaufgabe: Das neue Mobilitätskonzept für Wien 2025. Elektrotechnik und Informationstechnik, 134, 115-116.

Zimmermann, G. E. (2001). Räumliche Mobilität. In B. Schäfers \& W. Zapf (Hrsg.), Handwörterbuch zur Gesellschaft Deutschlands (S. 529-538). Wiesbaden: VS Verlag für Sozialwissenschaften.

Zoche, P., Kimpeler, M., \& Joepgen, M. (2002). Virtuelle Mobilität: Ein Phänomen mit physischen Konsequenzen? Zur Wirkung der Nutzung von Chat, Online-Banking und Online-Reiseangeboten auf das physische Mobilitätsverhalten. Berlin: Springer.

Open Access Dieses Kapitel wird unter der Creative Commons Namensnennung 4.0 International Lizenz (http://creativecommons.org/licenses/by/4.0/deed.de) veröffentlicht, welche die Nutzung, Vervielfältigung, Bearbeitung, Verbreitung und Wiedergabe in jeglichem Medium und Format erlaubt, sofern Sie den/die ursprünglichen Autor(en) und die Quelle ordnungsgemäß nennen, einen Link zur Creative Commons Lizenz beifügen und angeben, ob Änderungen vorgenommen wurden.

Die in diesem Kapitel enthaltenen Bilder und sonstiges Drittmaterial unterliegen ebenfalls der genannten Creative Commons Lizenz, sofern sich aus der Abbildungslegende nichts anderes ergibt. Sofern das betreffende Material nicht unter der genannten Creative Commons Lizenz steht und die betreffende Handlung nicht nach gesetzlichen Vorschriften erlaubt ist, ist für die oben aufgeführten Weiterverwendungen des Materials die Einwilligung des jeweiligen Rechteinhabers einzuholen.

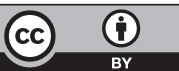

